\title{
Lactobacillus fermentum exerts a beneficial effect in an experimental model of rheumatoid arthritis in mice
}

\author{
M. E. Rodríguez-Cabezas ${ }^{1}$, F. Fisac ${ }^{1}$, E. Bailon ${ }^{1}$, M. Comalada ${ }^{1}$, D. Camuesco ${ }^{1}$, J. Xaus ${ }^{2}$, \\ A. Concha ${ }^{3}$, P. Talavera ${ }^{3}$, A. Nieto ${ }^{4}$, A. Zarzuelo ${ }^{1}$ and J. Galvez ${ }^{1}$ \\ ${ }^{1}$ Department of Pharmacology, University of Granada, Spain, ${ }^{2}$ Puleva Biotech SA, Granada, Spain, ${ }^{3}$ Department of \\ Pathology, Hospital Universitario 'Virgen de las Nieves', Granada, Spain and ${ }^{4}$ Health and Progress Foundation, \\ Granada, Spain
}

Previous studies have shown the intestinal anti-inflammatory activity of the probiotic Lactobacillus fermentum CECT 5716 acting as a modulator of the intestinal immune response ${ }^{(1)}$. The aim of the present study was to determine the efficacy of this probiotic as a systemic immune modulator in the experimental model of rheumatoid arthritis induced by Mycobacterium butyricum-Freund's adjuvant in mice ${ }^{(2)}$. Male Balb/C mice ( $n$ 10) were given the probiotic in the drinking water $\left(10^{8}\right.$ colony-forming units (CFU)/ml) for 2 weeks and a control group ( $n$ 20) not receiving probiotic was also used for reference. After this period $0.1 \mathrm{ml}$ of a suspension of $10 \mathrm{mg}$ Mycobacterium butyricum in $10 \mathrm{ml}$ Freund's adjuvant was administered subcutaneously in the right paw in treated mice and in half the control group, whereas Freund's adjuvant was administered in the left paw of the mice; normal mice $(n$ 10) only received Freund's adjuvant in both paws. Animals were assessed for clinical arthritis for 4 weeks, and after this period all mice were killed and their arthritic status evaluated by histological analysis of the paws. In addition, the spleens were removed and T-cells were obtained from spleens and activated with concanavalin A and the lymphocyte response evaluated by RT-PCR for the cytokines IL-2 and IL-10.

The results showed that the probiotic treatment ameliorated the severity of the disease since it significantly improved the clinical arthritis score (6.0 (SE 0.7) in control mice with arthritis v. 3.3 (SE 0.3) in probiotic-treated mice; $P<0.05)$ (Figure 1). Similar findings were obtained by histological analysis. Mycobacterium-induced arthritis also stimulated the expression of IL-2 in the splenocytes, which was also reduced in the probiotic-treated mice. Although IL-10 expression by splenic T-cells was not modified during the course of arthritis in control mice, treatment with the probiotic stimulated the expression of this regulatory cytokine in mice with arthritis. In conclusion, probiotic treatment ameliorated the disease outcome in the Mycobacterium butyricum model of rheumatoid arthritis in mice, showing systemic immunomodulatory properties.

Figure 1: CLINICAL ARTHRITIS SCORE

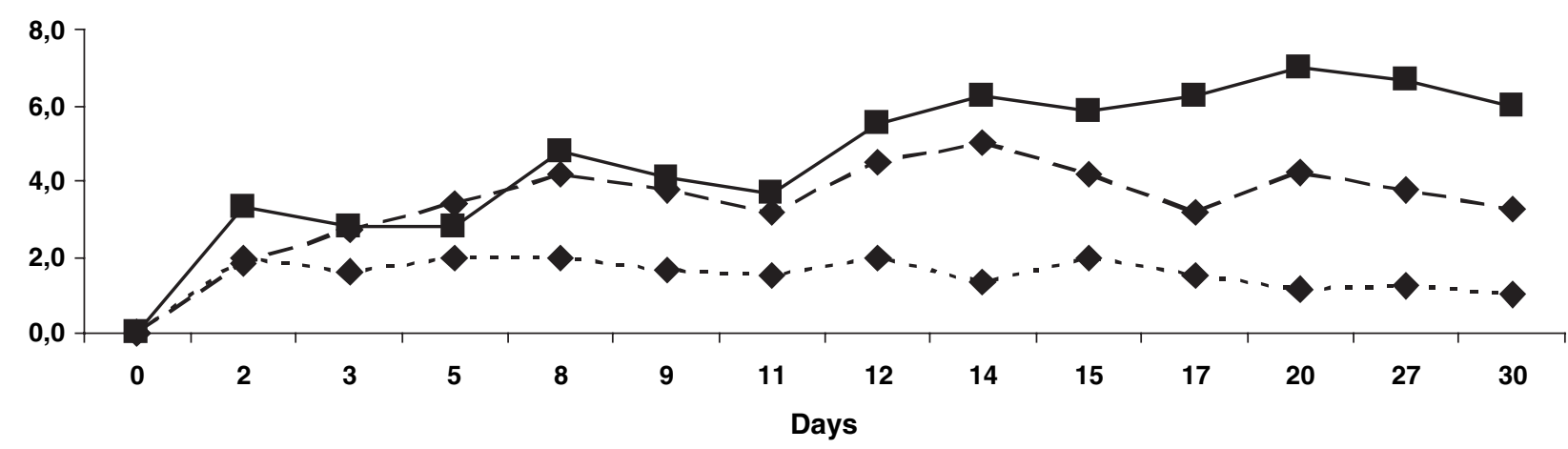

$-\bullet$ Non arthritic $\rightarrow-$ Control arthritic $\longrightarrow \bullet \cdot$ L.fermentum

1. Peran L, Camuesco D, Comalada M et al. (2006) Int J Colorectal Dis 21, 737-746.

2. Mihara M, Nishimoto N, Yoshizaki K \& Suzuki T (2002) Immunol Lett 84, 223-229. 